\title{
BILATERAL EXTERNAL ILIAC PSEUDOANEURYSMS CAUSING URINARY OBSTRUCTION AND ACUTE RENAL FAILURE
}

Marcelo Puppo Bigarella ( $\sim$ mpbiga@gmail.com )

University of Sao Paulo: Universidade de Sao Paulo https://orcid.org/0000-0002-7665-4794

Roberto Iglesias Lopes

University of Sao Paulo: Universidade de Sao Paulo

Guilherme Gentile

University of Sao Paulo: Universidade de Sao Paulo

Carolina Brito Faustino

University of Sao Paulo: Universidade de Sao Paulo

Lais da Cunha Gamba

University of Sao Paulo: Universidade de Sao Paulo

Guilherme Baumgardt Barbosa Lima

University of Sao Paulo: Universidade de Sao Paulo

Henry Augusto Hoffman Melo

University of Sao Paulo: Universidade de Sao Paulo

Grace Carvajal Mulatti

University of Sao Paulo: Universidade de Sao Paulo

\section{Research Article}

Keywords: Ruptured pseudoaneurysm, External iliac artery, Ureteral obstruction, Bilateral ureterohydronephrosis, Endovascular repair

Posted Date: January 25th, 2022

DOl: https://doi.org/10.21203/rs.3.rs-512207/v1

License: (c) (i) This work is licensed under a Creative Commons Attribution 4.0 International License.

Read Full License 


\section{Abstract}

Background: Pseudoaneurysms are usually caused by trauma, infections, vasculitis, atherosclerosis or iatrogenic complications $[1,2]$. Frequently, these pseudoaneurysms remain clinically silent, but few can become symptomatic and might present with limb ischemia, leg edema, as well as subacute or acute hemorrhage. An unusual presentation of a bilateral external iliac artery (EIA) pseudoaneurysm is here reported.

Case presentation: We report an exceedingly rare case of a young man presenting an acute severe bilateral testicular pain radiating to the back. Clinical and radiological work-up demonstrated bilateral EIA pseudoaneurysms that caused bilateral ureterohydronephrosis due to the urinary obstruction with subsequent renal failure. Management included immediate bilateral external iliac endovascular repair and bilateral ureterolysis through a retroperitoneal approach, with resolution of the obstruction and successful endovascular treatment of both pseudoaneurysms. The only identifiable risk factor for cardiovascular disease was cocaine addiction.

Conclusion: Uncommon and severe clinical presentation of bilateral EIA pseudoaneurysms caused bilateral ureterohydronephrosis and acute renal failure. Although the presenting symptoms were deceiving at first, fast diagnosis might have been crucial in this case, in which there was a overlapping among Urology and Vascular Surgery - both specialties involved in treating the patient. Awareness of this condition might prevent misdiagnosis and delays in management that are of utmost importance for a good outcome.

\section{Background}

Bilateral ureteral obstruction and acute kidney injury (AKI) due to bilateral iliac artery conditions is a very rare entity, with few reported cases $[3,4]$. This observation might be explained by the reported prevalence of this condition. Opposite to the association of iliac artery aneurysms and an abdominal aortic aneurysm which is relatively common, isolated iliac artery aneurysms are rare $(0.4$ to $1.9 \%$ of all intraabdominal aneurysms) [5], with only $10 \%$ arising from EIA [6]. To our knowledge, this is the first reported case of EIA pseudoaneurysms that caused bilateral ureterohydronephrosis with subsequent renal failure.

\section{Case Presentation}

A 29-year old man was admitted to the emergency department presenting an acute severe bilateral testicular pain radiating to the back. The pain initiated during night, awakening the patient and prompting medical evaluation. He detailed the pain as sharp and continuous, irradiating to his lower back bilaterally and without worsening or alleviating factors. No history of trauma, underlying medical conditions, regular medications or previous surgeries was reported. Abnormal urinary symptoms were also ruled out.

At admission, he was slightly hypotensive $(110 / 60 \mathrm{mmHg})$ with a normal heart rate and normal neurological status. Physical examination was remarkable for a mild dehydration, with pale mucous 
membranes and cool extremities. Peripheral pulse was symmetric in lower and upper limbs. No heart murmurs, signs of pulmonary congestion or pleural effusion were observed. On abdominal palpation, a lower abdominal mass was noted with mild discomfort. No signs of acute peritonitis were present. Testicular exam was unremarkable.

Anemia (hemoglobin level of $7.6 \mathrm{~g} / \mathrm{dl}$ and hematocrit of 24\%) and acute kidney failure was observed (creatinine $2.8 \mathrm{~g} / \mathrm{dl}$, normal blood urea with a estimated Glomerular Filtration Rate of $29 \mathrm{~mL} / \mathrm{min} / 1.73$ $\mathrm{m}^{2}$ ). Testicular tumors markers were negative. Abdominal ultrasonography revealed a retroperitoneal mass causing bilateral ureterohydronephrosis. Testes imaging was completely normal.

Contrast-enhanced multiaxial tomography (CT) scan of abdomen and pelvis was deemed necessary, even in the setting of AKI, to establish the definitive diagnosis. It revealed bilateral EIA pseudoaneurysms (Figure 1A) causing moderate bilateral ureterohydronephrosis (Figure 1B) with clear signs of contrast leakage from the right EIA pseudoaneurysm into the retroperitoneum, an evidence of an acute ruptured pseudoaneurysm associated with an ipsilateral retroperitoneal hematoma (Figure 1C). These findings justified acute anemia and renal failure.

The patient was blood-typed and saline expansion was performed. A central left jugular vein catheter was placed in case of acute deterioration and on-call vascular surgery performed a bedside Doppler ultrasonography of the iliac arteries confirming the bilateral enlargement with a turbulent pattern (in comparison to distal or aortic smooth laminar pattern) on the right EIA, thereby confirming the findings of a ruptured right iliac pseudoaneurysm (Figure 2).

Due to the risks of an ongoing bleeding, the patient was submitted to an emergency endovascular repair of the bilateral EIA pseudoaneuryms (Figures 3A, 3B and 3C), ruptured on the right side (as shown in Figure 3D) by the application of bilateral $8 \mathrm{~mm}$ (in diameter) $\times 50 \mathrm{~mm}$ (in length) WALLGRAFT ${ }^{\circledR}$ endoprosthesis (Boston Scientific, Marlborough, Massachusetts, United States). After angiographic confirmation of successful treatment with no further bleeding or leakage (Figure 3E and 3F), open inguinotomies were performed for ureterolysis and drainage of retroperitoneal hematoma.

Immediate post-decompression diuresis was observed (with an impressive volume of 12 liters of diuresis on the first 24 hours following the procedure). Creatinine peaked at $3.2 \mathrm{~g} / \mathrm{dl}$ on the first postoperative day, with the patient reaching a normalized renal function after a week of follow-up. During this period, patient was closely monitored for his hemodynamic condition and renal function recovery, preventing dehydration and electrolyte imbalances.

Hematoma content evacuated from perianeurysmal region as well as conjunctive tissue resected were sent to histopathological analysis and tested for bacteria and fungus. Serologies for acquired infectious disease, inflammatory exams and other specific rheumatological tests (antinuclear factor for lupus and other autoimmune tests) were performed and a multidisciplinary panel of Vascular Surgery, Internal Medicine, Infectious Disease, Rheumatology and Urology fully evaluated the patient. Results were within 
normal range and cultures were negative. The only identifiable risk factor for cardiovascular disease was cocaine addiction (for the last 2 years).

\section{Conclusions}

An uncommon clinical presentation of an acute severe bilateral testicular pain radiating to the back, a compensated hypovolemic shock, acute anemia and AKI were the most remarkable early findings of the clinical presentation. Common underlying causes of pseudoaneurysms were excluded, with the only identifiable risk factor for cardiovascular disease the patient's cocaine addiction. This clinical presentation is worrisome, and we believe it merits the report of such a case as a misdiagnosis would probably result in significant delay of treatment in a critical emergency condition. Hypovolemic shock in case of rupture might occur, therefore immediate management is crucial, either with an open or with an endovascular approach. Endovascular intervention using a covered stent has the advantages of being less invasive and faster control of bleeding is achieved in most cases [7].

In our case, alleviation of urinary obstruction was also necessary. Hematoma drainage through an open retroperitoneal approach and bilateral ureterolysis were performed in order to prevent infection and to release the ureteral encasement caused by these inflammatory pseudoaneurysms. This management strategy seems more effective than only ureteral stenting in order to prevent hematoma infection and promote early renal function recovery.

This case highlights the uncommon and severe clinical presentation of bilateral EIApseudoaneurysms causing bilateral ureterohydronephrosis and subsequent renal failure. Radiological work-up showed a ruptured pseudoaneurysm on the right side although the patient was still on the compensated hypovolemic shock phase. Awareness of this condition might prevent misdiagnosis and delays in management that are of utmost importance for a good outcome.

\section{Abbreviations}

Contrast-enhanced multiaxial tomography (CT)

acute kidney injury (AKI)

bilateral external iliac artery (EIA)

\section{Declarations}

Consent for publication

All procedures performed in studies were in accordance with the ethical standards of the institutional and/or national research committees and with the 1964 Helsinki Declaration and its later amendments or 
comparable ethical standards. This study has obtained IRB approval from Hospital das Clínicas - USP and the need for informed consent was waived. The authors declare that they have no conflict of interest. This study was not supported by any funding.

Availability of data and material

Not applicable

Competing interests

The authors declare that they have no competing interests

Funding

None

Authors' contributions

MPB is the main author and wrote the manuscript. RIL is the second author, reviewed the entire manuscript, gave guidance to the project and helped with the submission. GG retrieved personal information from the patient from the hospital databank, as well other exams. CBF, LCG, HAHM were at the surgery, and provided all imaging material for the report. GBBL and GCM were responsible for reviewing the vascular accuracy of the report.

Acknowledgements

We could not have carried out this case report without the dedicated involvement and valuable scientific contribution of Prof. William Carlos Nahas and Prof. Miguel Srougi (Urology), as well Prof. Pedro PuechLeão and Prof. Nelson de Luccia (Vascular Surgery).

\section{References}

1. Sueyoshi E, Sakamoto I, Nakashima K, Minami K, Hayashi K. Visceral and peripheral arterial pseudoaneurysms. Am J Roentgenol. Published online 2005. doi:10.2214/ajr.185.3.01850741

2. Lazarides $M K$, Arvanitis $D P$, Dayantas $J N$. latrogenic arterial trauma associated with hip joint surgery: An overview. Eur J Vasc Surg. Published online 1991. doi:10.1016/S0950-821X(05)80343-3

3. Wu Z, Ma Y. Acute kidney injury secondary to bilateral common iliac artery aneurysms. Intern Med J. Published online 2019. doi:10.1111/imj.14225

4. Oliveira $\mathrm{M}$, Sousa $\mathrm{C}$, Coentrão L. Bilateral common iliac artery aneurysms as cause of renal failure. Intensive Care Med. Published online 2018. doi:10.1007/s00134-017-4936-4

5. Richardson JW, Greenfield LJ. Natural history and management of iliac aneurysms. J Vasc Surg. Published online 1988. doi:10.1016/0741-5214(88)90405-3 
6. Krupski WG, Selzman $\mathrm{CH}$, Floridia R, et al. Contemporary management of isolated iliac aneurysms. $J$ Vasc Surg. Published online 1998. doi:10.1016/S0741-5214(98)70194-6

7. Adovasio R, Mucelli FP, Lubrano G, et al. Endovascular treatment of external iliac artery injuries after hip arthroplasty. J Endovasc Ther. Published online 2003. doi:10.1583/1545-

1550(2003)010<0672:ETOEIA>2.0.CO,2

\section{Figures}

\section{Figure 1}

Contrast enhanced CT-Scan of Abdomen and Pelvis A) axial view, showing bilateral pseudoaneurysms of EIA with large diameter, B) axial view, demonstrating bilateral ureterohydronephrosis, C) Coronal view, in which leakage of contrast is seen in the right side.

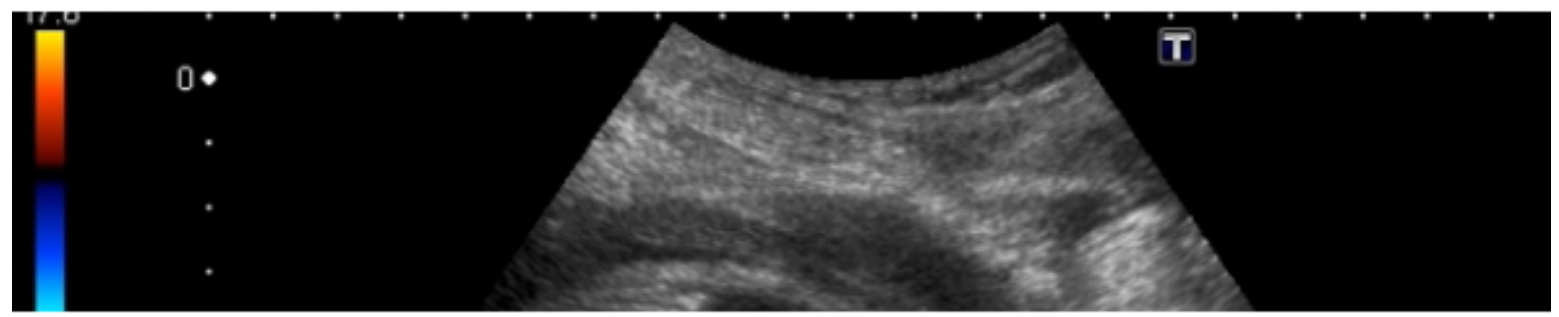

\section{Figure 2}

Doppler Ultrasound of right EIA, demonstrating $10.6 \times 9.4 \mathrm{~cm}$ pseudoaneurysm with with turbulent flow to the posterior iliopsoas region, compatible with rupture 


\section{Figure 3}
A) 3D pre-operative vessel reconstruction, B) Intra-operative arteriography of iliac arteries, C) Selective arteriography of left EIA pseudoaneurysm, D) Selective arteriography of right EIA pseudoaneurysm, showing contrast leakage - left endoprosthesis is already seen E) Arteriography of left side after treatment, F) Control arteriography for the right side, now without contrast leakage. 